Recepción: 20 / 04 / 2017

Aceptación: 20 / 05 / 2017

Publicación: 15 / 07 / 2017
Ciencias Naturales Artículo Científico

\title{
Determinación de Taninos y Cumarinas presente en la planta tres filos
}

\section{Determination of Tanins and Cumarines present in the three philosophy plant}

\section{Determinação de Taninos e Cumarínicos presentes na planta três gumes}

Ingrid A. Herrera-Fuentes I
ingrid.herreraf16@gmail.com

Katty L. Quimis-Ponce II

lizpunk94@hotmail.com

Nancy A. Sorroza-Rojas III

nancysorroza@uees.edu.ec

\author{
Frella S. García-Larreta IV \\ soraya.garcial@ug.edu.ec \\ Walter Mariscal-Santi VI \\ walter.mariscals@ug.edu.ec \\ Raisa S. Mariscal-García VI \\ raisamariscal@hotmail.com
}

Correspondencia: ingrid.herreraf16@gmail.com

\footnotetext{
${ }^{\mathrm{I}}$ Docente, Universidad de Guayaquil, Guayaquil, Ecuador.

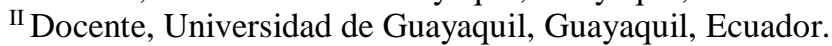

${ }^{\mathrm{III}}$ Docente, Universidad de Especialidades Espíritu Santo, Samborondón, Ecuador.

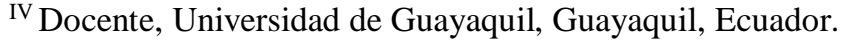

${ }^{\vee}$ Docente, Universidad de Guayaquil, Guayaquil, Ecuador.

${ }^{\mathrm{VI}}$ Docente, Universidad de Guayaquil, Guayaquil, Ecuador.
} 


\section{Resumen}

En esta investigación se abordó la cuantificación de Taninos y Cumarinas presentes en la Planta Tres Filos (Baccharis genistelloides). Los ejemplares trabajados fueron recolectados en la Parroquia Mariscal Sucre del Cantón Milagro, se seleccionaron las hojas como material de trabajo tomando en cuenta los criterios de inclusión y exclusión. Se llevaron a cabo pruebas analíticas para su análisis, tales como: pureza, humedad, cenizas totales, cenizas solubles en agua, cenizas insolubles en ácido clorhídrico. Se obtuvieron los extractos metanólico, etanólico, y acuoso. Se realizó un tamizaje fitoquímico para determinar la concentración de Taninos y Cumarinas. Como resultado se apreció que de acuerdo a una clasificación de mayor (+++) a menor (+) cantidad presente frente a un patrón seleccionado, la cuantificación de la concentración para Taninos en el extracto etanólico fue (+++), en el extracto metanólico (++), en el extracto acuoso (+). Para Cumarinas, se obtuvo en el extracto acuoso (+), para el extracto metanólico (++), para el extracto etanólico (+++). En general, en el extracto etanólico se obtuvo una mayor concentración de Taninos y Cumarinas. Se encontró bibliográficamente que las concentraciones de Taninos y Cumarinas de diferentes especies del género Baccharis diferían. Para concluir lo anterior, se relacionaron resultados del análisis de los extractos hidroalcohólicos realizados por otros autores en B. latifolia, B. salicifolia, B. glutinosa. Las concentraciones de los extractos en las tres distintas especies de Baccharis estudiadas con respecto a $B$. genistelloides, mostraron que tienen los mismos metabolitos secundarios, pero en distintas concentraciones. Los resultados comparativos fueron: B. latifolia: Taninos (+), Cumarinas (-), B. salicifolia: Taninos (+++), Cumarinas (+++), B. glutinosa: Taninos (+++), Cumarinas (+++), B. genistelloides: Taninos (+++), Cumarinas (+++). Como recomendación una profundización del estudio (B. genistelloides), dado que dentro de la población donde se recolectó la muestra, esta planta se usa en diferentes aplicaciones terapéuticas tradicionales.

Palabras Claves: Taninos; cumarinas; Baccharis genistelloides; concentración; etnobotanica, 


\section{Summary}

In this research the quantification of Tannins and Coumarins present in the Plant Three Filos (Baccharis genistelloides) was approached. The specimens worked were collected in the Parish Mariscal Sucre del Cantón Milagro, the leaves were selected as work material taking into account the inclusion and exclusion criteria. Analytical tests were carried out for analysis, such as: purity, humidity, total ash, water-soluble ash, insoluble ash in hydrochloric acid. The methanolic, ethanolic, and aqueous extracts were obtained. A phytochemical screening was performed to determine the concentration of tannins and coumarins. As a result it was observed that according to a classification of greater (+++) to lower $(+)$ amount present against a selected standard, the quantification of the concentration for Tannins in the ethanolic extract was (+++), in the Methanolic extract (++), in the aqueous extract (+). For coumarins, the aqueous extract $(+)$ was obtained for the methanolic extract $(++)$, for the ethanolic extract (+++). In general, in the ethanolic extract a higher concentration of tannins and coumarins was obtained. It was found bibliographically that the concentrations of Tannins and Coumarins of different species of the genus Baccharis differed. To conclude the above, results of the analysis of the hydroalcoholic extracts made by other authors in B. latifolia, B. salicifolia, and B. glutinosa were related. The concentrations of the extracts in the three different species of Baccharis studied with respect to B. genistelloides, showed that they have the same secondary metabolites, but in different concentrations. Comparative results were: B. latifolia: Tannins (+), Cumarinas (-), B. salicifolia: Tannins (+++), Coumarins (+++), B. glutinosa: Tannins $(+++)$, Coumarins +++$)$, B. genistelloides: Tannins (+++), Coumarins (+++). As a recommendation, a deepening of the study (B. genistelloides), given that within the population where the sample was collected, this plant is used in different traditional therapeutic applications.

Key Words: Tannins; coumarins; Baccharis genistelloides; concentration; ethnobotanics. 


\section{Resumo}

Nesta pesquisa quantificar Taninos e cumarinas presentes na planta Três Filos (Baccharis genistelloides) foi abordada. Os espécimes trabalhados foram coletadas na Paróquia Marechal Sucre Miracle Canton, eles saem como material de trabalho tendo em conta os critérios de inclusão e exclusão foram selecionados. pureza, humidade, cinzas totais, cinzas solúvel em água, cinza insolúvel em ácido clorídrico: ensaios analíticos para a análise, tal como foram realizadas. Obtiveram-se os extractos metanólicos, etanólicas e aquosas. Um rastreio fitoquímico foi realizada para determinar a concentração de taninos e cumarinas. Como resultado observou-se que de acordo com uma classificação de mais elevado $(+++)$ para baixa quantidade (+) presentes antes de um padrão seleccionado, quantificando o extracto de etanol de concentração foi Taninos (+++), sobre o extracto metanólico (++) no extracto aquoso (+). Para cumarinas, que foi obtido com o extracto aquoso (+), para o extracto de metanol (++), para o extracto etanólico (+++). Em geral, no etanólico extrair uma maior concentração de taninos e cumarinas foi obtido. Bibliograficamente descobriram que concentrações de taninos e cumarinas espécies diferentes Baccharis diferiu. Para concluir os resultados da análise acima extratos hidroalcoólicos feitas por outros autores em B. latifolia, B. salicifolia, B. glutinosa eles relacionados. As concentrações dos extractos em três espécies diferentes de Baccharis estudados no que diz respeito a B. genistelloides mostrou que tem os mesmos metabolitos secundários, mas em diferentes concentrações. Os resultados comparativos foram: B. latifolia: Taninos (+), cumarinas (-), B. Salicifolia: Taninos (+++), cumarinas (+++), B. glutinosa: taninos $(+++)$, cumarinas $(+++)$, B. genistelloides: taninos $(+++)$, cumarinas $(+++)$. Como recomendação estudo aprofundamento (B. genistelloides), uma vez que na população em que a amostra foi colhida, a planta é usada em várias aplicações terapêuticas tradicionais.

Palavras-chave: Taninos; cumarinas; Baccharis genistelloides; concentração; etnobotânica. 
Ingrid A. Herrera-Fuentes; Katty L. Quimis-Ponce; Nancy A. Sorroza-Rojas; Frella S. García-Larreta; Walter Mariscal-Santi;

\section{Introducción.}

Las plantas son una importante fuente de productos biológicamente activos, muchos de los cuales han servido como modelo para la síntesis de un gran grupo de fármacos, mundialmente se estima que cerca del $25 \%$ de todos los medicamentos modernos son derivados de plantas medicinales. (Jara Beltrán, 2013)

Las plantas con propiedades medicinales han estado relacionadas estrechamente con el surgimiento y desarrollo de la civilización humana en diferentes zonas geográficas y han sido reconocidas por la OMS, para el tratamiento de diversas enfermedades, el Ecuador, se caracteriza por ser uno de los países, a nivel mundial, con mayor biodiversidad y riqueza en conocimientos ancestrales relacionados con el uso de las plantas medicinales. (Bilbao, 1997) (Aguirre \& Belmares, 2012)

Aprovechando que en Ecuador existe una gran diversidad de plantas de las cuales muchas no han sido reconocidas, ni estudiadas, resulta de gran importancia y compromiso investigar sobre las especies vegetales, sobre todo aquellas que no son tan conocidas y quiza poco valoradas por el desconocimiento de sus virtudes y han sido utlizadas en la medicina tradicional. (Gonzalez \& Peña, 2001)

La parroquia rural Mariscal Sucre está ubicada en el cantón Milagro que pertenece a la Provincia del Guayas, en dicha parroquia las personas que sufren heridas por objetos que son usados en las labores diarias de la agricultura, hacen uso recurrente de la planta llamada comúnmente Tres Filos. 
La planta Tres Filos cuyo nombre científico es (Baccharis genistelloides), es denominada una planta medicinal silvestre, comúnmente utilizada como anestésico y para los dolores estomacales.

Se distribuye en Colombia, Ecuador, Perú, Bolivia y Chile. En Ecuador se encuentra ampliamente expandida entre los 2000 y los 4500 metros sobre el nivel del mar.

Resulta importante reconocer la concentracion de taninos y cumarinas presentes en la planta los cuales intervienen en la coagulacion sanguínea.

\section{Metodología}

\section{Medicina tradicional}

Es todo el conjunto de conocimientos, aptitudes y prácticas basados en teorías, creencias y experiencias indígenas de las diferentes culturas, sean o no explicables, usados para el mantenimiento de la salud, así como para la prevención, el diagnóstico, la mejora o el tratamiento de enfermedades físicas o mentales. (OMS, 2016)

\section{Medicina tradicional herbolaria: La medicina herbolaria es la utilización de plantas y extractos de hierbas por su valor terapéutico.}

\section{Materiales herbario:}

Comprenden, además de hierbas, jugos frescos, gomas, aceites fijos, aceites esenciales, resinas y polvos secos de hierbas. En algunos países esos productos se pueden elaborar mediante diversos procedimientos locales, como el tratamiento con vapor, el tostado o el rehogado con miel, bebidas alcohólicas u otros materiales. (OMS, 2016) 
Ingrid A. Herrera-Fuentes; Katty L. Quimis-Ponce; Nancy A. Sorroza-Rojas; Frella S. García-Larreta; Walter Mariscal-Santi;

\section{Droga}

Se define como todo producto de origen natural que recolectado o separado de su medio tiene una composición y unas propiedades tales dentro de su complejidad que constituye la forma bruta de un medicamento.

\section{Familia Asteraceae}

Es reconocida por el hombre como plantas alimenticias y/o medicinales, esta es la más numerosa de todas las fanerógamas con aproximadamente 23.000 especies y más de 1.500 géneros distribuidas por todo el mundo.

\section{Genero Baccharis}

Es el género más rico en especies dentro de la familia Asteraceae, está compuesto por más de 400-500 especies distribuidas principalmente en regiones tropicales del continente como: Brasil, Argentina, Colombia, Chile y México. Su distribución es exclusivamente Americana, va desde el Sur de los Estados Unidos hasta el extremo austral de Chile y Argentina.

En otros lugares del continente, diversas especies de este género son también utilizadas tradicionalmente para el tratamiento de varias dolencias, tales como contusiones, inflamaciones, también como antimicrobiano y desinfectante, lo cual indica la enorme potencialidad que las plantas de este género poseen y que permiten poner de manifiesto un escenario claro para futuros estudios que lleven, por un lado, a la validación de tales usos, y, por el otro, a proporcionar, dentro de estudios adicionales de bioprospección, un valor agregado a este género de plantas tan versátiles. 
Un ejemplo claro está en Brasil, es usada en la medicina tradicional para el control y tratamiento de diferentes enfermedades, la consumen principalmente cómo té, para aliviar dolencias de estómago, el hígado, inflamaciones, anemia, diabetes, desintoxicación del cuerpo y también para enfermedades de la próstata.

\section{Propiedades Terapéuticas}

Es usada para trastornos gastrointestinales, enfermedades reumáticas, fiebre, diabetes, problemas de hígado, cicatrizante de heridas, quemaduras, llagas y ulceraciones.

\section{Taninos}

No es fácil dar una definición de este grupo de compuestos naturales que tienen una gran distribución en la naturaleza, ya que el término incluye sustancias que se agrupan por la analogía estructural, sino por características comunes.

El término tanino fue originalmente utilizado para describir ciertas sustancias orgánicas que servían para convertir a las pieles crudas de animales en cuero, proceso conocido en inglés como tanning ("curtido" en español). Los taninos tienen un ligero olor característico, sabor amargo y astringente, y su color va desde el amarillo hasta el castaño oscuro.

Etimológicamente el término tanino se refiere al poder para curtir la piel de un animal y convertirla en un cuero flexible, estable y resistente a la putrefacción.

Son metabolitos polifenólicos ampliamente distribuidos en el reino vegetal. Se localizan en todas las partes de las plantas y su concentración es variable a lo largo del ciclo vegetativo. En la medicina tradicional se usan externamente, los preparados a base de drogas ricas en taninos, como 
Ingrid A. Herrera-Fuentes; Katty L. Quimis-Ponce; Nancy A. Sorroza-Rojas; Frella S. García-Larreta; Walter Mariscal-Santi;

las decocciones, se emplean para detener pequeñas hemorragias locales; en inflamaciones de la cavidad bucal, catarros, bronquitis, quemaduras, hemorroides. poder antiséptico para prevenir germinación de hongos y crecimiento de parásitos.

\section{Clasificación.}

Dada su diversidad química y estructural, estos compuestos suelen clasificarse como taninos hidrolizables y taninos condensados.

\section{Taninos hidrolizables}

Los taninos hidrolizables son moléculas polifenólicas de tamaño discreto, de naturaleza no polimérica, capaces de ser hidrolizados por ácidos o enzimas a un azúcar y a un ácido carboxílico fenólico.

Corresponden al parecer, a los de mayor distribución en el reino vegetal, estructuralmente son ésteres de diferentes ácidos fenólicos, por lo que son, en realidad, mezclas des estructuras que hacen complejas su constitución. Se caracterizan por ser muy solubles en agua, sobre todo en agua caliente, formando soluciones colidales. Son sólidos amorfos muy higroscópicos de color amarillo pardusco.

Cuando están relativamente puros, disminuyen su solublidad en agua y son más cristalinos. Son, por tanto, también más solubles en solventes orgánicos de relativa polaridad, en particular el metanol y el etanol. 
Los que se encuentran en forma glicosídica se pueden hidrolizar parcial o totalmente durante el proceso de extracción y se precipitan con facilidad de las soluciones donde se hallan, por adición de ácidos minerales. De ellos se diferencian dos tipos.

Galotaninos: Estructuralmente, son ésteres del ácido gálico con la glucosa o un polisacárido, o la variante derivada del ácido metadigálico.

Elagitaninos: Son taninos que producen por hidrólisis tanto el ácido gálico, como algunos de sus derivados. No necesariamente aparecen fusionados en sus orígenes a la glucosa, sino que pueden aparecer después de la hidrólisis, por la regeneración de los agrupamientos lactónicos. Agregan las estructuras del ácido elágico e hidrogálico. (Miranda \& Cuellar, 2012)

\section{Taninos condensados}

Los taninos condensados son moléculas oligoméricas o poliméricas de unidades flavan-3-ol, unidas a través de enlaces C-4 a C-8, o menos frecuentemente, C-4 a C-6 de dos unidades consecutivas de diverso tamaño y constitución según su origen, además son resistentes a la hidrólisis.

También presentan mucha dispersión en el reino vegetal, y se considera como característica fundamental, que producen antocianidinas y catequinas pro calentamiento con ácidos diluidos. Se condesan con proteínas cuya estructura depende lógicamente de la masa molecular. De ellos se reconocen tres tipos.

Catequinas (flavan-3-oles): Se encuentran, por lo general, libres en la naturaleza y responden estructuralmente a las variantes: catequina y galacotaquina. 
Ingrid A. Herrera-Fuentes; Katty L. Quimis-Ponce; Nancy A. Sorroza-Rojas; Frella S. García-Larreta; Walter Mariscal-Santi;

Los miembros más comunes sólo difieren del número de $\mathrm{OH}$ en el anillo $\mathrm{B}$, los cuales no se han encontrado metilados. Se ha demostrado que el hidroxilo en la posición 3, se une por puente de hidrógeno con el oxígeno heterocíclico.

Leucoanatocianidinas (flavan-3,4-dioles): Se diferencian de las catequinas en que estas producen productos insolubles, de color amarillo oscuro, cuando se calientan con ácidos; mientras los flavn-3,4- dioles producen, además de esto, flobafenos y algunas antocianidinas.

\section{Aplicaciones}

Tanto los taninos hidrolizables y condensados, se emplean en la industria del cuero, por su gran poder curtiente, permitiendo obtener una amplia variedad de cueros, que se diferencian en flexibilidad y resistencia.

Los taninos condensados se usan principalmente en la fabricación de adhesivos y resinas. Por ejemplo, algunos tipos de adhesivos y resinas taninos han servido para desarrollar adhesivos en frío y termofraguados por tratamiento con úreaformaldehído, o con copolímeros fenolformaldehído; estos últimos usados en la fabricación de enchapes de madera a prueba de agua.

Los taninos hidrolizables encuentran amplia aplicación debido a sus propiedades antioxidantes y su habilidad para formar complejos solubles e insolubles con las proteínas.

Se usan como estabilizadores de la cerveza: en el producto que no ha sido recientemente preparado, las proteínas se combinan con los polifenoles para formar complejos que son responsables de la presencia de turbidez, al agregar los taninos, el nivel de proteínas disminuye a un valor apropiado y se aumenta así el tiempo de almacenamiento de la cerveza. 
En la industria farmacéutica, se emplean para contraatacar el efecto de los alcaloides y el envenenamiento por sales de metales, inactivándose éstos por precipitación.

En la industria de alimentos se puede, por ejemplo, eliminar impurezas proteínicas por precipitación con taninos, emplearlos en la preservación y maduración de alimentos, aprovechando sus propiedades antisépticas y antioxidantes, y también en la clarificación del vino blanco.

En cuanto a los extractos de taninos se señala, el efecto cariostático (inhibidor de caries), el efecto inhibidor para el crecimiento de diversos virus y la actividad hemostática y antiinflamatoria en formulaciones basadas en taninos.

\section{Cumarinas}

Las cumarinas fueron aisladas y purificadas por primera vez por Voleg, en 1822, a partir del haba tonka (Dypteryx odorata), y deben su denominación a la palabra "coumarou", nombre vernáculo de esta haba.

Las Cumarinas son lactonas insaturadas, cuyo heteroátomo es el oxígeno, son otra clase de compuestos orgánicos del tipo $\mathrm{C}_{6} \mathrm{C}_{3}$, cuyo heteronúcleo se denomina benzo 2-pirona o benzo $\alpha$ pirona y su formación es clave en la síntesis de ciclación de toda cumarina

Las cumarinas pertenecen al grupo de los metabolitos secundarios sintetizados por la acción del ácido shikímico mediante el metabolito intermedio de la función metabólica del ácido

\section{Características}

Soluble: soluciones acuosas o alcohólicas de hidróxido de sodio. 
Ingrid A. Herrera-Fuentes; Katty L. Quimis-Ponce; Nancy A. Sorroza-Rojas; Frella S. García-Larreta; Walter Mariscal-Santi;

Presentan un espectro UV característico, influido fuertemente por la naturaleza y posición de los sustituyentes, que se modifica profundamente en medio alcalino. A la luz ultravioleta, las cumarinas presentan fluorescencia variable, de azul a amarillo y a púrpura, exaltada en presencia de amoniaco.

La mayoría de las cumarinas conocidas, se encuentran libres en las plantas y con frecuencia en cualquiera de los órganos vegetales, desde raíces hasta flores y frutos, son sustancias fluorescentes, comúnmente fotosensibles.

Aunque presentan una amplia distribución en el reino vegetal, hay familias donde se encuentran con mayor probabilidad, siendo estas las siguientes: Leguminosas, Orchidáceas, Rutáceas Y Umbelíferas.

Las cumarinas biosintetizadas en las plantas son almacenadas en el interior de las vacuolas al igual que los alcoloides, los taninos y los lignanos

\section{Clasificación}

a) Cumarinas Simples: Presentan derivados oxigenados en las posiciones 6,7 y 8 del núcleo bencénico y en las posiciones 3 y 4 del núcleo lactona. Son los derivados 8 hidroxilados, alcoxilados y alquilados) y glicósidos de las 1,2 -Benzopirona. En la naturaleza, un 95\% de las cumarinas poseen un radical oxigenado en la posición 7

b) Cumarinas complejas: Se dividen en furanocumarinas y piranocumarinas, por la posición de fusión del anillo furano y del anillo pirano. Esta división se subclasifica en lineales. 
c) Cumarinas diversas: Son cumarinas que presentan las variaciones de las cumarinas complejas en el anillo piranósico y furanósico. Se denominan cumarinas preliminares por la presencia de sustituyentes en el anillo pirano o furano

\section{Extracción}

Las cumarinas son solubles en alcohol, se extraen con disolventes orgánicos como el éter.

Las formas heterosídicas son más o menos solubles en agua.

\section{Actividad biológica de las cumarinas}

Debido a la gran variedad estructural de estas moléculas son muchas las propiedades farmacológicas asociadas a dicho anillo, entre otras: antimicrobianas, antiinflamatorias, antiespasmódicas, antivirales, antihelmínticas, antioxidantes, o inhibidoras enzimáticas

La warfarina y el carbocromeno, ambos utilizados actualmente en clínica, son dos conocidos ejemplos de la familia de las cumarinas con actividad cardioprotectora, debido a su acción vasodilatadora e inhibidora de la agregación plaquetaria

Las furanocumarinas tienen la propiedad de ser fotosensibilizadoras de la piel; es usada en el tratamiento de algunas alteraciones de la piel como por ejemplo para el tratamiento de psoriasis. Las piranocumarinas tienen acción antiespasmódica y vasodilatadora de las arterias coronarias, propiedades estrogénicas, acción antiinflamatoria, antibacteriana, hipotérmica y anticoagulante.

Además de la importancia en farmacognosia, hay que destacar también las aplicaciones de las cumarinas en las que actúan como saborizantes, aromatizante, aditivos alimentarios y en perfumería. 
Ingrid A. Herrera-Fuentes; Katty L. Quimis-Ponce; Nancy A. Sorroza-Rojas; Frella S. García-Larreta; Walter Mariscal-Santi;

Preparación de la muestra

Recolección de las hojas de la planta (Baccharis genistelloides) en La Parroquia Mariscal

Sucre, Cantón - Milagro.

\section{Criterios de Inclusión y exclusión}

a) Inclusión: Hojas frescas, adultas, y del tamaño adecuado. Dimensiones $5.5 \mathrm{~cm}$ de largo, $3 \mathrm{~cm}$ de ancho, y verdes brillantes.

b) Exclusión: Hojas pequeñas que no han llegado a la adultez y menores al tamaño. Hojas ennegrecidas, hojas amarillentas quemadas por el sol.

Pruebas analíticas

\section{Humedad}

Llevar al horno secador para extraer la humedad de las mismas a $60^{\circ} \mathrm{C}$ durante 4 horas.

Porcentaje de humedad de la muestra:

$\% \mathrm{H}=(\mathrm{p}-\mathrm{p} ") / \mathrm{p} \times 100$

$\mathrm{p}=$ peso inicial de la muestra

p"= peso final de la muestra

\section{Pureza}

a) Peso total de la droga (pt): Pesar toda la droga, eliminar el peso del envase. 
b) Determinación de hojas ennegrecidas (He): Se procede manualmente a separar las hojas ennegrecidas y se pesa anotando la misma.

c) Determinación de partes no apropiadas de la propia planta $(\mathrm{Pp})$ : Se procede a separar manualmente partes no apropiadas de la propia planta, que se estime no están en buenas condiciones o que no intervienen como principio medicinal (tallos, hojas, flores, raíces.) se pesa y se anota su valor.

d) Determinación de materia orgánica extraña (Mo): Se procede a separar hojas, tallos, semillas, etc., que no pertenecen a la droga cruda analizada. Se pesa y se registra su valor.

e) Determinación de materia inorgánica extraña (Mi): Procedemos a separar las partículas de tierra, arena y piedrecillas presentes en muestra. Pesar y registrar su valor.

f) Determinación de la pureza de la droga cruda (P): El peso total de la droga (pt) corresponde al $100 \%$

$\% \mathrm{P}=(\mathrm{He}+\mathrm{Pp}+\mathrm{Mo}+\mathrm{Mi}) / \mathrm{PT} \times 100$

\section{Cenizas totales}

Con la ayuda de 2 crisoles previamente tarados, pesamos 2 gr. de muestra vegetal seca y molida en cada crisol, y llevamos a la mufla por el lapso de 3 horas a $750^{\circ} \mathrm{C}$, pasado este tiempo dejar en secador y pesar.

$\mathrm{C}=\frac{M 2-M}{M 1-M} X 100$

M2 = Masa de crisol con cenizas 
Ingrid A. Herrera-Fuentes; Katty L. Quimis-Ponce; Nancy A. Sorroza-Rojas; Frella S. García-Larreta; Walter Mariscal-Santi;

M1 = Masa de crisol con muestra de ensayo

M = Masa de crisol vacío

\section{Cenizas Totales en Agua}

Pesar $2 \mathrm{~g}$ de muestra vegetal seca y molida en dos crisoles respectivamente, llevar a la Estufa por 3 horas a $750^{\circ} \mathrm{C}$, pasado este tiempo dejar en secador y pesar. (Fernández, 2014)

M2 = Masa de crisol con cenizas

M1 = Masa de crisol con muestra de ensayo

M = Masa de crisol vacío

$\mathrm{C}=\frac{M 2-M}{M 1-M} X 100$

Cenizas Totales en Ácido Clorhídrico.

En el crisol con ceniza colocar $2 \mathrm{ml}$ de $\mathrm{HCl}$ al $10 \%$, tapar el crisol con papel aluminio, poner agua a hervir previamente en un vaso de precipitación, posteriormente procedemos a filtrar el contenido del crisol con papel filtro tarado, con la ayuda del agua caliente realizamos varios enjuagues al papel filtro con residuo descartando el líquido del filtrado, en la último enjuague tomamos una muestra el líquido y le adicionamos 1 gota de $\mathrm{HNO}_{3}$ y de 1-2 gotas de $\mathrm{AgNO}_{3}$, si se obtuviese un color blanco en el líquido se procederá a seguir los enjuagues del papel filtro con agua caliente hasta que el líquido al adicionarle las gotas de reactivo no tomasen ningún color es decir queden transparente. Luego de esto poner el papel filtro con residuo en el crisol correspondiente para llevarlo a la mufla por 2 horas. A $750^{\circ} \mathrm{C}$. 
Preparación y Obtención de extractos.

Estos se obtienen mediante la separación de porciones biológicamente activas presentes en los tejidos de las plantas, con el uso de un solvente (alcohol, agua, mezcla de estos u otro solvente selectivo) y un proceso de extracción adecuado.

Extracto etéreo.

Pesar 25 g. de droga seca y molida y extraerlos con 200 ml de éter etílico por maceración durante 48 horas. Se obtiene el extracto y el residuo sólido (secar y pesar).

\section{Extracto metanólico}

Identificación de Cumarinas - Técnica Oliveros Et. Al. 2011.

Para la construcción de una curva de calibración se preparó una solución madre (25mL) de cumarina patrón de concentración 1,9 mM. Luego se tomaron volúmenes en forma creciente y se fueron añadiendo a matraces de $10 \mathrm{~mL}$ que luego fueron llevados al aforo con el solvente. De esta manera construyeron soluciones patrón de cumarina de 5, 10, 50, 100, 300, 500, 800 y $1000 \mu \mathrm{M}$. Se tomó directamente de estas soluciones patrones para analizar en el HPLC. Cada determinación se llevó a cabo por triplicado.

Equipos usados: Estufa (Memert) modelo SN-B 20, Balanza Analítica Koaten, Espectrofotómetro Genesys, HPLC-UV (Agilent) modelo L-1120

\section{Resultados.}

En base a lo realizado se determinó el parámetro de pureza en las hojas es fundamental para garantizar la idoneidad de la muestra. 
Ingrid A. Herrera-Fuentes; Katty L. Quimis-Ponce; Nancy A. Sorroza-Rojas; Frella S. García-Larreta; Walter Mariscal-Santi;

\section{Determinación de Pureza}

\section{(Resultados de Pureza Baccharis genistelloides)}

\begin{tabular}{|l|l|}
\hline Impureza & $24.5 \%$ \\
\hline Pureza & $75.5 \%$ \\
\hline
\end{tabular}

Fuente (Herrera \& Quimis, 2017)

Para la identificación organoléptica de (Baccharis genistelloides) se tomaron en cuenta los criterios de inclusión y exclusión, obteniendo como resultado un $75.5 \%$ de pureza y un $24.5 \%$ impureza, lo cual permite determinar que las hojas califican para el procedimiento analítico posterior a la identificación de Taninos y Cumarinas.

\begin{tabular}{|l|l|l|l|}
\hline Muestras & Peso Inicial & Peso Final & $\%$ \\
\hline
\end{tabular}

\section{Tamizaje Fitoquímico}

Se realizó el estudio fitoquímico de los extractos acuoso, metanólico, etanólico de (Baccharis genistelloides) para aislar, cuantificar Taninos y Cumarinas, obteniendo los siguientes resultados. 


\section{(Resultados Tamizaje Fitoquímico Baccharis genistelloides)}

\begin{tabular}{|l|l|l|l|}
\hline METABOLITOS SECUNDARIOS & Acuoso & Etanólico & Metanólico \\
\hline TANINOS & + & +++ & ++ \\
\hline CUMARINAS & + & +++ & ++ \\
\hline
\end{tabular}

Fuente (Herrera \& Quimis, 2017)

Ausencia: (-). Evidencia Baja: (+). Evidencia Media: (++). Evidencia Alta: (+++).

De acuerdo a los resultados en la determinación fitoquímica de (Baccharis genistelloides), se evidenció la presencia de una baja concentración de Taninos y Cumarinas en el extracto acuoso ya que los Taninos y Cumarinas son compuestos polares y el agua es no polar, lo que impide la unión de puentes de hidrogeno.

Mientras que en el extracto metanólico se obtuvo una concentración media y en el extracto etanólico una concentración alta, esto se debe a que se prefiere que sean solventes polares como el éter y el metanol lo que permiten la solubilidad de los Taninos y Cumarinas para una mayor cuantificación en el análisis.

Según el estudio comparativo del tamizaje fitoquímico de (Baccharis genistelloides) VS los tamizajes fitoquímicos de tres diferentes especies, Baccharis latifolia, Baccharis salicifolia, Baccharis glutinosa en el extracto hidroalcohólico, se evidencia las concentraciones de Taninos y 
Ingrid A. Herrera-Fuentes; Katty L. Quimis-Ponce; Nancy A. Sorroza-Rojas; Frella S. García-Larreta; Walter Mariscal-Santi; Raisa S. Mariscal-García

Cumarinas ya que la Familia Asteraceae tiene una abundancia de los metabolitos secundarios en las especies de Baccharis comparadas.

Análisis cuantitativo en extracto acuoso, extracto etanólico, extracto metanólico

(Resultados Análisis cuantitativo en extracto acuoso, extracto etanólico, extracto

metanólico Baccharis genistelloides)

\begin{tabular}{|l|l|l|l|}
\hline METABOLITOS SECUNDARIOS & Acuoso & Etanólico & Metanólico \\
\hline TANINOS & $0.20 \%$ & $<1.0 \mathrm{mg} / \mathrm{Kg}$ & $0.16 \%$ \\
& & & \\
\hline CUMARINAS & $1.04 \%$ & $184.25 \mathrm{mg} / \mathrm{Kg}$ & $448.46 \mathrm{mg} / \mathrm{Kg}$ \\
\hline
\end{tabular}

Fuente (Herrera \& Quimis, 2017)

Se cuantifico Taninos obteniéndose en el extracto acuoso $0.20 \%$, extracto etanólico $<1.0$ $\mathrm{mg} 7 \mathrm{Kg}$, extracto metanólico $0.16 \%$. Para Cumarinas en el extracto acuoso $1.04 \%$, extracto etanólico $184.25 \mathrm{mg} / \mathrm{Kg}$, extracto metanólico $448.46 \mathrm{mg} / \mathrm{Kg}$.

\section{Conclusiones.}

Los parámetros físico-químicos analizados de la planta Tres Filos (Baccharis genistelloides) encontrados fueron: Pureza 75.5\%, Impureza 24.5\%, Humedad 54\%-72\%, Cenizas Totales $3.42 \%$, Cenizas Solubles en agua 1.13\%, Cenizas Insolubles en $\mathrm{HCl}$ 0.75\% estos se ubican dentro de los parámetros de la Farmacopea.

Con el Tamizaje fitoquímico en los extractos acuosos, metanolicos y etanólicos se pudo aislar y cuantificar los metabolitos secundarios Taninos y Cumarinas de la planta Tres Filos 
(Baccharis genistelloides), obteniendo como resultado la presencia de una baja concentración de Taninos en el extracto acuoso (+), mientras que en el extracto metanólico una moderada concentración $(++)$, y en el extracto etanólico una concentración alta (+++). Para Cumarinas en el extracto acuoso (+), mientras que en el extracto metanólico (++), y en el extracto etanólico una concentración alta $(+++)$

Se relacionaron los extractos hidroalcohólicos de tres especies de Baccharis que contenían Taninos y Cumarinas VS (Baccharis genistelloides), teniendo como resultado de la comparación para Baccharis latifolia en Taninos una concentración baja(+), para la concentración de Cumarinas una ausencia (-), Baccharis salicifolia en Taninos una concentración alta (+++), así mismo para Cumarinas una concentración alta (+++), Baccharis glutinosa presento Taninos con una concentración alta $(+++)$, también para Cumarinas una concentración alta $(+++)$.

Tanto Baccharis genistelloides, Baccharis salicifolia, Baccharis glutionosa presenta para Taninos una alta concentración (+++), así mismo para Cumarinas una alta concentración (+++).

Cabe recalcar que durante esta investigación de la planta Tres Filos (Baccharis genistelloides) no se encontró ningún estudio que detalle la concentración de los metabolitos secundarios Taninos y Cumarinas de esta especie de Baccharis, siendo estos datos los primeros propuestos.

\section{Recomendaciones}

Se recomienda la profundización de las caracterizaciones morfológicas y etnobotánicas de esta especie (Baccharis genistelloides). 
Ingrid A. Herrera-Fuentes; Katty L. Quimis-Ponce; Nancy A. Sorroza-Rojas; Frella S. García-Larreta; Walter Mariscal-Santi; Raisa S. Mariscal-García

Analizar el resto de metabolitos presentes en (Baccharis genistelloides) para profundizar estudios de sus propiedades terapéuticas.

Realzar los beneficios de la medicina tradicional ya que dentro de la población donde se recolectó la muestra, esta planta es usada en diferentes aplicaciones terapéuticas.

\section{Bibliografía.}

Aguirre, J., \& Belmares, R. (2012). Actividad antioxidante de algunas plantas tropicales, subtropicales y semideserticas. Revista cientifica de la Universidad Autonona de Coahuila.

Bilbao, M. (1997). Analisis Fitoquimico Preliminar. Quimica de Productos Naturales. Armenia: Universidad del Quindio.

Fernández, D. (2014). Obtenido de Estudio de acción hipoglucemiante y desinflamatoria de la chilca (Baccharis latifolia) en la provincia de El: http://repositorio.utmachala.edu.ec/bitstream/48000/1354/7/CD00258-TESIS.pdf

Gonzalez, Y., \& Peña, M. (2001). Taninos de diferentes especies vegetales en la prevencion del fotoenvejecimiento. Revista Cubana de Investigaciones Biomedicas, 20(1).

Jara Beltrán, A. (2013). Analisis fitoquímico y determinación de la actividad antioxidante del extracto etanólico de las hojas de la especie Piper imperiale (Piperaceae). Bogotá: Universidad de Ciencias Aplicadas y Ambientales.

Miranda, M., \& Cuellar, A. (2012). Farmacognosia y Productos Naturales. Guayaquil, Guayas, Ecuador: Felix Varela.

OMS. (2016). Medicina tradicional: definiciones. Ginebra: Organmizacion Mundial de la Salud . 